\title{
Extramedullary Plasmacytoma Of The Nasal Cavity
}

\section{S Kanotra, M Lateef}

\section{Citation}

S Kanotra, M Lateef. Extramedullary Plasmacytoma Of The Nasal Cavity. The Internet Journal of Otorhinolaryngology. 2009 Volume 11 Number 2.

DOI: $\underline{10.5580 / 1827}$

\begin{abstract}
Extramedullary Plasmacytomas(EMP) are rare tumors accounting for $0.4 \%$ of all head and neck malignancies and $4 \%$ of all nonepithelial tumors of nasal cavity. The absence of multiple myeloma must be confirmed with bone marrow examination and skeletal surveys, before arriving at a diagnosis of EMP. We report a case of extramedullary plasmacytoma of the nasal cavity in a 60 year old female, who was a chronic tobacco sniffer. The case demonstrates the multidisciplinary approach required for the optimal diagnosis and management of such tumors and proposes chronic irritation of the nasal mucosa as one of the etiological factors for EMP.
\end{abstract}

\section{INTRODUCTION}

Extramedullary Plasmacytomas(EMP) are rare tumors accounting for $0.4 \%$ of all head and neck malignancies and $4 \%$ of all nonepithelial tumors of nasal cavity. ${ }^{1}$ About $80 \%$ of the extramedullary plasmacytomas are localized in the submucosa of the upper respiratory tract, with a large proportion occurring in the sinonasal/nasopharyngeal area. ${ }^{1-3}$ The absence of multiple myeloma must be confirmed with bone marrow examination and skeletal surveys ,before arriving at a diagnosis of EMP. The therapy consists of combined surgery and radiotherapy. The main prognostic indicator for EMP is progression to multiple myeloma.10-32\% of all patients develop multiple myeloma which reduces the mean survival time from 8.3 years to 20 months ${ }^{1,4}$ We report a case of EMP of the nasal cavity in a 60 year old female who was a chronic tobacco sniffer. The patient was managed by endoscopic removal of the tumor followed by radiotherapy. A 6 month follow up of the patient revealed neither recurrence of the tumor nor a progression to multiple myeloma.

\section{CASE REPORT}

A 60 year old female, with unremarkable past medical history, presented with right sided nasal obstruction, mucoid nasal discharge and intermittent epistaxis for the past 5 months. There was history of occasional headaches. The patient had been sniffing tobacco for the past 35 years. The general physical examination did not reveal any abnormality, nor was there any evidence of lymphadenopathy. Anterior rhinoscopy revealed a pinkish, fleshy mass in the right nasal cavity which was firm in consistency, bled easily and was insensitive to probing. Endoscopic examination confirmed the presence of a dark red sub mucosal lesion with a smooth surface. The CT scan of the patient revealed a hypodense mass localized to the right nasal cavity showing contrast enhancement with no bony erosion (figure 1 ).A nasal biopsy was subsequently taken which revealed a dense infiltrate of plasma cells ( figure 2 ). A histological diagnosis of plasmacytoma was made. Routine blood tests and serum biochemistry including calcium, phosphorus, blood urea nitrogen, urea, uric acid and creatinine were all within reference range. Both serum myeloma proteins and urine Bence- Jones proteins were negative. Serum and urine electrophoresis did not reveal $\mathrm{M}$ component. Bone marrow biopsy revealed a plasma cell infiltrate of less than $3 \%$ of all nucleated cells .Chest radiograph, total body skeletal survey and Tc99 scintigram, showed no systemic lesion. The finding confirmed a diagnosis of stage 1 extramedullary plasmacytoma. The patient underwent complete surgical removal of the tumor endoscopically. This was followed by radiotherapy of $5000 \mathrm{cGy}$, delivered over a period of 6 weeks. A 6 month follow- up of the patient did not reveal any recurrence. 


\section{Figure 1}

FIGURE:1: CONTRAST ENHANCED CORONAL CT SCAN PNS SHOWING HYPODENSE MASS IN THE RIGHT NASAL CAVITY.

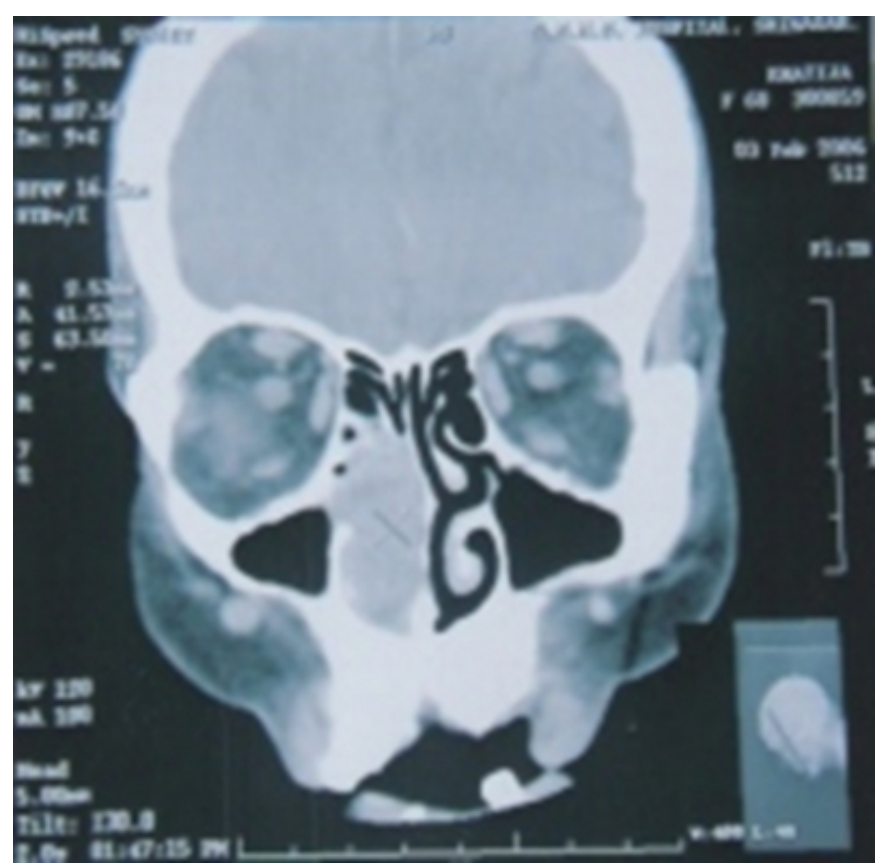

\section{Figure 2}

FIGURE 2: MICROPICTOGRAPH SHOWING SHEETS OF CLOSELY PACKED PLASMA CELLS

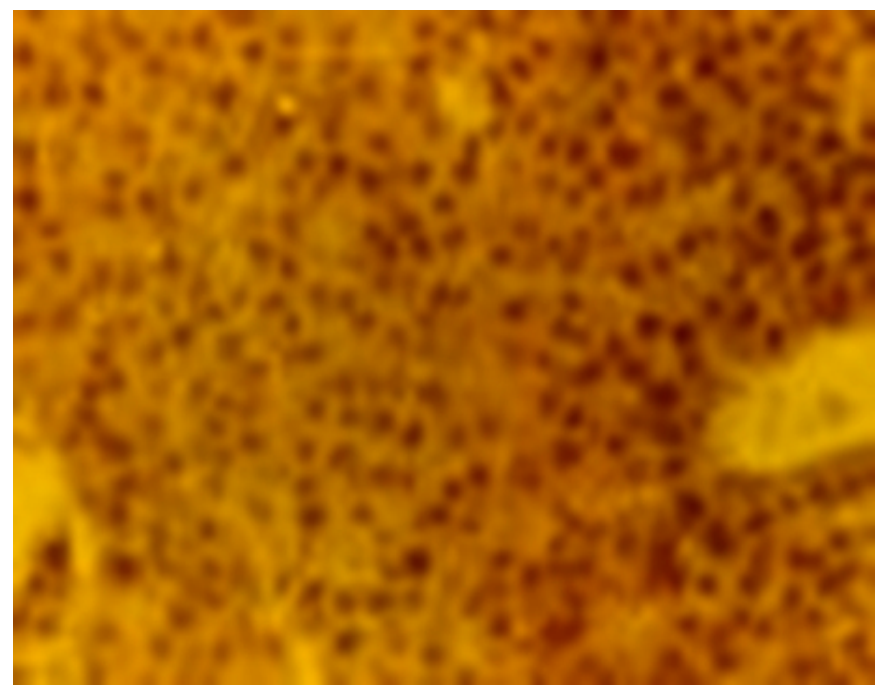

\section{DISCUSSION}

Plasma cell neoplasm are a group of clinical disorders characterized by monoclonal expansion of plasma cells that elaborate a single homogenous immunoglobulin molecule or fragment. Depending on their site of development and clinical features, plasma cell neoplasm are divided into: a) Solitary extramedullary plasmacytoma (b) Solitary bone plasmacytoma (c) Multiple myeloma (d)Multifocal form of multiple myeloma (e) Plasmablastic sarcoma. ${ }^{5}$

Extramedullary Plasmacytoma is a localized collection of monoclonal plasma cells, arising within soft tissues in an extraskeletal site. Extramedullary Plasmacytomas can either be - Primary (true) Plasmacytoma (without evidence of systemic disease) or Extramedullary manifestation during the course of multiple myeloma.

The first case of EMP was reported by Schridde in $1905 .{ }^{6}$ Alexiou et al in 1999, reviewed all previous reports of EMP and found 869 cases out of which 714(82.2\%) had occurred in the upper aero digestive tract.The most commonly affected area in this region were the nasal cavity/Para nasal sinuses $(43.8 \%)$ followed by nasopharynx $(18.3 \%)$, the oropharynx $(17.8 \%)$ and $\operatorname{larynx}(11.1 \%){ }^{5}$

EMP affects males 3-4 times more than females with a median age of 55 years (range 50-65 years) which is 10 years younger than multiple myeloma. ${ }^{7}$ Most of the EMP's are solitary lesions, however $10-20 \%$ manifest as multiple lesions. ${ }^{8}$ The etiology of EMP is still unknown. Because of its presentation in the mucosa of the upper aero digestive tract (78\%), chronic stimulation by inhaled irritants or viral infection has been implicated as causative factors. ${ }^{2}$ The clinical presentation varies according to the site of involvement. As most lesions are located in the sinonasal/nasopharyngeal area, the presenting symptoms include soft tissue mass or swelling (80\%),airway obstruction (35\%), epistaxis (35\%),pain (20\%), proptosis $(15 \%)$, nasal discharge (10\%), regional lymphadenopathy (10\%), or cranial nerve palsy (5\%) with the median duration of symptoms being 4-5 months. ${ }^{7.9}$ The physical examination essentially reveals a gray to red, soft or firm, sessile or pedunculated, submucosal mass that bleeds easily and is usually nontender and smooth without obvious ulceration of the mucosa. Osseous destruction is uncommon in extramedullary plasmacytomas.

Histopathologically, the tumor is characterized by a dense homogenous infiltrate of plasma cells with varying degree of dysplasia. The plasma cells have round eccentric nuclei with dense chromatin clumps arranged along the nuclear membrane in a cartwheel fashion. Plasmacytic, plasmablastic and anaplastic cell types have been described. Local amyloid deposits are found in 11-38\% of cases; systemic amyloid being very rare. No amyloid was detected in our patient. Histopathology cannot distinguish multiple myeloma from extramedullary plasmacytoma. Confirmation of diagnosis can only be made after any systemic 
involvement has been excluded. A complete blood count with WBC count and platelet count, serum biochemistry including calcium, BUN, creatinine, uric acid, serum proteins, serum and urine electrophoresis, bone marrow biopsy and a complete skeletal survey are recommended to rule out multiple myeloma. ${ }^{10}$

The diagnostic criterion for EMP include: (a) Biopsy of tissue showing monoclonal plasma cell histology. (b)Bone marrow plasma cell infiltrates not exceeding $5 \%$ of all nucleated cells. (c) Absence of osteolytic bone lesions or other tissue involvement (no evidence of myeloma elsewhere). (d) Absence of hypercalcemia or renal failure. (e) Low serum M protein concentration, if present. ${ }^{11}$

EMP can be staged as follows: Stage 1-Limited to extramedullary site. Stage 2: Local extension or involvement of regional lymph nodes. Stage 3: Disseminated disease. ${ }^{2}$

According to Batsakis, the natural history of extramedullary plasmacytoma may be characterized as: (a) localized, solitary controlled by surgery, radiotherapy, or both, does not recur or become disseminated.(b) locally recurrent controlled by additional therapy.(c)aggressive, persistent or recurrent disease, producing death through uncontrollable local extensions.(d) local disease with "metastatic" involvement of regional lymph nodes without evidence of distant spread.(e) local disease, recurrent or otherwise followed by dissemination and development of multiple plasma cell neoplasm and/or multiple myeloma. ${ }^{12}$

The treatment of Extramedullary Plasmacytoma is still controversial. Some clinicians favour radiotherapy ${ }^{2}$, others prefer surgical excision, while some advocate combined approach. ${ }^{13}$.Alexiou et al in their review of 714 cases of upper aero digestive tract extramedullary plasmacytomas reported between 1905-1997, found that the median overall survival or recurrence free survival was longer than 300 months for patients who underwent combined surgery and radiotherapy as compared to a median survival rate of 144 months for patients who underwent radiotherapy and 156 months for surgically managed patients. ${ }^{5}$ Most clinicians recommend a combined approach ( surgery and radiotherapy ) for the management of upper aero digestive EMP. ${ }^{2,13}$ The optimal dose for local control is $5000 \mathrm{cGy}$, with a fraction size of 200 cGy delivered over 4-6 weeks. ${ }^{5}$ The patient in our case had localized disease of the nasal cavity which was resected surgically and subsequently subjected to radiotherapy.

The median survival of patients varies from 4-10 years. Local recurrences have been reported in $22 \%$ cases of adequately treated EMP in the upper aero digestive tract with $16 \%$ of EMP evolving subsequently into multiple myeloma. ${ }^{5}$ Because of the tendency of EMP to progress into disseminated multiple myeloma, a lifelong follow-up of these patients is recommended.

Our case clearly demonstrates that a multidisciplinary approach is required for the optimal diagnosis and management of extramedullary plasmacytoma. It is essential to exclude any systemic involvement before arriving at a diagnosis of solitary extramedullary plasmacytoma. The present case being a chronic tobacco sniffer, further supports Witshaw's view that chronic stimulation of the mucosa of the upper aero digestive tract by inhaled irritants may be one of the etiological factors for EMP. ${ }^{2}$

\section{References}

1. Fu YS, Perzin KH . Non epithelial tumors of nasal cavity, paranasal sinuses and nasopharynx. Cancer 1978; 42: 2399-2406.

2. Wiltshaw $\mathrm{E}$. The natural history of extramedullary plasmacytoma and its relation to solitary myeloma of bone and myelomatosis . Medicine 1976; 55:217-238

3. Wax MK, Yun KJ, Omar RA. Extramedullary Plasmacytomas of the head and neck. Otolaryngol Head Neck Surgery 1993;109:877-885

4. Kyle RA .Multiple myeloma .Review of 869 cases. Mayo Clinic Proceedings 1975;50: 29-40.

5. Alexiou C, Kau RJ, Dietzfelbinger $\mathrm{H}$, et al:

Extramedullary Plasmacytoma : tumor occurrence and therapeutic concepts. Cancer 1999 Jun 1; 85(11): 2305-14

6 . Schridde $H$. Weitere Untersuchungen uber die kornelungen der plasmazellen. Centralbl Allg Pathol Anat.1905 ; 16:433-435.

7. Kapadia SB, Desai U, Cheng VS. Extramedullary Plasmacytoma of head and neck. A clinicopathological study of 20 cases .Medicine 1982;61:317-319.

8. Batsakin JG, Medriros JL, Luna MA, El-Naggar AK

.Plasma cell dyscrasias and the head and neck. Ann Diagn Pathol 2002;6:129-140

9. Miller FR, Lavertu P, Wanamaker JR et al.

Plasmacytomas of head and neck. Otolaryngol Head Neck Surg. 1998;119:614-618.

10. Abemayor E, Canalis RF, Greenberg P, Wortham DG,

Rowland JP , Sun NC. Plasma cell tumors of head and neck .J Otolaryngol 1988; 17: 376-381.

11. Galieni P, Cavo M, Pulsoni A, et al ; Clinical outcomes of extramedullary plasmacytoma. Haematologica 2000;85 :47-51.

12. Batsakis JG.Plasma cell tumors of head and neck. Ann Otol Rhinol Laryngol. 1992; 92:311-313.

13. Castro EB, Lewis JS, Strong EW. Plasmacytomas of Paranasal sinuses and nasal cavity. Arch Otolaryngol 1973 ;97:326-329. 


\section{Author Information}

Sohit Paul Kanotra, (MS)

Post Graduate Scholar, Department Of Ent, Head \& Neck Surgery, Government Medical College

Mohammad Lateef, (MS)

Professor \& Head, Department Of Ent, Head \& Neck Surgery, Government Medical College 